\title{
Diuréticos del asa y ototoxicidad
}

\author{
Martínez-Rodríguez R, García Lorenzo J*, Bellido Peti J, Palou Redorta J, Gómez Ruiz JJ, \\ Villavicencio Mavrich $\mathrm{H}$.
}

Servicio Urología Fundación Puigvert, Barcelona. *Servicio otorrinolaringología Hospital Sant Pau, Barcelona.

Actas Urol Esp. 2007;31(10):1189-1192

\section{RESUMEN}

\section{DIURÉTICOS DEL ASA Y OTOTOXICIDAD}

El uso de diuréticos del asa esta muy extendido en la práctica medica. Con un amplio margen de seguridad, poseen efectos adversos que deben ser tenidos en cuenta para evitarlos o reconocerlos en cuanto aparecen. La ototoxicidad asociada a la furosemida es, en principio, excepcional y remite con la supresión del tratamiento, aunque se ha descrito cofosis permanente por lesión coclear.

Palabras clave: Diuréticos del asa. Ototoxicidad. Yatrogenia.

\section{ABSTRACT}

\section{LOOP DIURETICS AND OTOTOXICITY}

Loop diuretics are quite often used in medical practice. In spite of a wide security, there are several adverse events that should be known, in order to be early recognised as soon as they appear. Ototoxicity associated to furosemide is infrequent and reversible. However, permanent deafness associated with furosemide administration has been previously reported.

Keywords: Loop diuretics. Ototoxicity. Yatrogenic.

$\mathrm{L}^{\mathrm{a}}$ a yatrogenia farmacológica puede alcanzar una prevalencia de hasta el 10\% en estudios transversales ${ }^{10}$. La sordera neurosensorial súbita es atribuida a infecciones víricas o bacterianas, fracturas de hueso temporal, enfermedades autoinmunes, neurológicas o vasculares, tumores y síndromes paraneoplásicos y el uso de fármacos ototóxicos ${ }^{1}$. Una correcta investigación de los posibles factores causales resulta determinante en el abordaje terapéutico y aumenta considerablemente las posibilidades de curación.

\section{CASO CLÍNICo}

Mujer de 62 años con antecedentes de hipertensión arterial, hipercolesterolemia e hipertrigliceridemia. Antecedente urológico de tumor vesical infiltrante de alto grado (Carcinoma de células transicionales G3T2 p53+ y Carcinoma in situ en muestra 1 de la biopsia múltiple normatizada).
Tras cistectomía radical con reconstrucción vesical tipo Studer (Carcinoma urotelial G3 de patrón sólido, pt3b, ganglios negativos) presenta sepsis de origen urinario ingresando en unidad de semicríticos con tratamiento antibiótico y diurético intensivos.

A los tres días del ingreso la paciente refiere acúfenos de instauración súbita acompañados de hipoacusia bilateral de grado importante. La paciente no presentaba clínica vertiginosa ni de inestabilidad. No había otalgia ni otodinia ni sensación de plenitud ótica. La movilidad facial estaba conservada.

La exploración física es estrictamente normal revelando la audiometría hipoacusia profunda del oído derecho y cofosis de oído izquierdo, con impedanciometría normal (Fig. 1).

Una vez confirmado el carácter neurosensorial de la hipoacusia se realizó una revisión de la historia clinica de la paciente y una nueva anamne- 


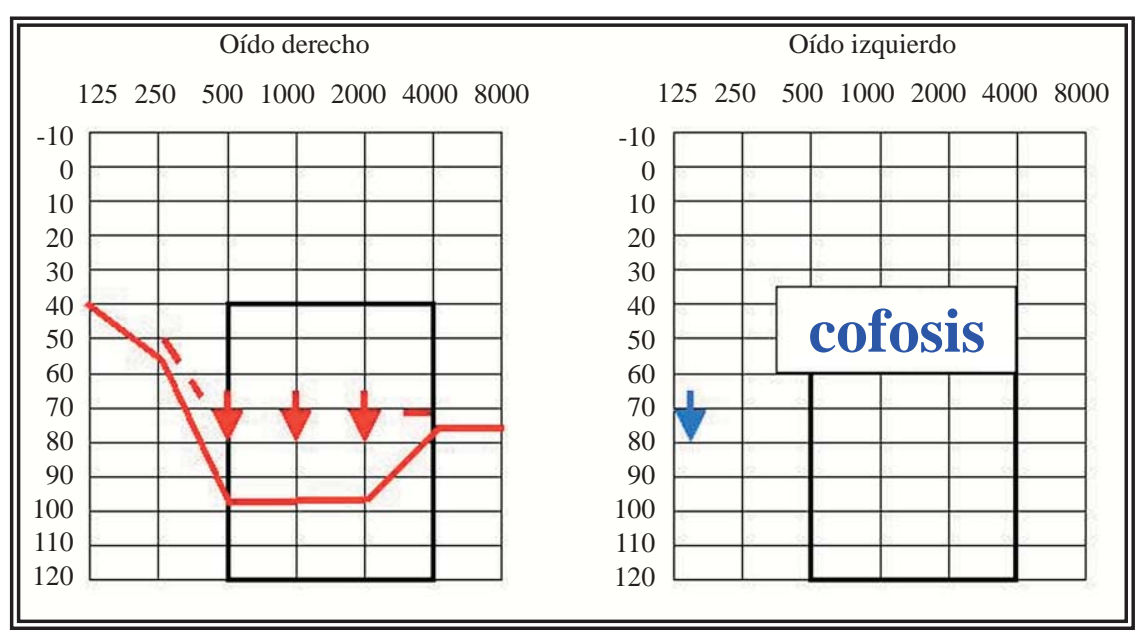

FIGURA 1. Audiometría al diagnóstico Oido derecho: 99,2\% y Oído izquierdo cofosis (Tablas AMA).

sis para realizar el estudio etiológico. Descartada la relación con enfermedades sistémicas, infecciones intracraneales y traumatismos, se atribuyó la hipoacusia a la administración de furosemida intravenosa en el manejo inicial del shock séptico.

Se suspendió la administración de furosemida, y se proscribió la asociación de otros fármacos ototóxicos. Se procedió según el protocolo de hipoacúsia súbita a tratamiento con corticoides parenterales a altas dosis (Metilprednisolona $120 \mathrm{mg} / 24)$ y pentoxifilina por vía oral. (400mg/ 8 h). Tras 7 días de tratamiento parenteral se continuó el tratamiento corticoideo por vía oral en pauta descendente durante 14 días más (Prednisona $60 \mathrm{mg} /$ día 7 días y 30mg/día 7días), manteniendo la pentoxifilina a la misma dosis durante varios meses. La audiometría a las dos semanas del tratamiento evidenciaba mejoría de la audición tonal con aparición de restos auditivos en graves en el oído izquierdo y el ascenso de los umbrales en oído derecho (Fig. 2).

A los 6 meses del tratamiento la paciente ha recuperado la audición del oído derecho hasta un $58 \%$. El oído izquierdo tiene restos auditivos en frecuencias graves, pero no se ha observado una recuperación manifiesta. La paciente sigue controles en nuestro centro, y se le ha recomendado la adaptación de una prótesis acústica en oído derecho. Oncológicamente ha iniciado tratamiento complementario con quimioterapia, sin afectación de sus niveles auditivos (Fig. 3).

\section{DISCUSIÓN}

La hipoacusia súbita es una entidad infrecuente, aunque no rara en la consulta del ORL. Se estima que hay unos 10 casos por cada 100.000 habitantes $y$ año, siendo habitualmente unilaterales (4-17\% bilaterales) ${ }^{1}$. El diagnóstico etiológico comprende un amplísimo abanico de causas que comprende enfermedades otológicas, sistémicas, tumores, traumas acústicos... que condicionan el abordaje terapéutico y el pronóstico de recuperación en cada caso. En nuestro caso una minuciosa investigación de antecedentes personales y familiares, y una revisión cuidadosa del historial clínico de la paciente nos llevaron al diagnóstico de hipoacusia de causa ototóxica, que se confirmó con la mejoría rápida tras la supresión del la furosemida y el tratamiento de soporte.

Múltiples medicamentos han sido relacionados, directa o indirectamente, con afectación del sistema coclear y/o vestibular (Tabla 1).

La ototoxicidad por diuréticos del asa ha sido reproducida experimentalmente en animales de laboratorio $^{2,3}$. Son varios los diuréticos relacionados con la afectación del oído interno: Furosemida, Ácido etacrinico, Bumetanida, Piretanida, Torasemida.

Existe cierta controversia sobre los efectos secundarios de la furosemida sobre el desarrollo neonatal del sistema auditivo en animales de laboratorio y en humanos ${ }^{4,5}$.

En los pocos casos descritos de ototoxicidad asociada al uso de furosemida esta se ha vista potenciada par la utilización de dosis altas administradas en cortos periodos de tiempo, el uso junto a otros fármacos ototóxicos, la patología de base del paciente (insuficiencia renal, hepática, cardiaca y alteraciones del equilibrio hidroelectrolítico) y la administración a niños prematuros. Se trata de una toxicidad de predominio coclear (frecuencias altas y medias), súbita y de recuperación rápida tras supresión del tratamiento; aunque hay algunos casos descritos de afectación permanente ${ }^{6}$; Raramente afecta al sistema 


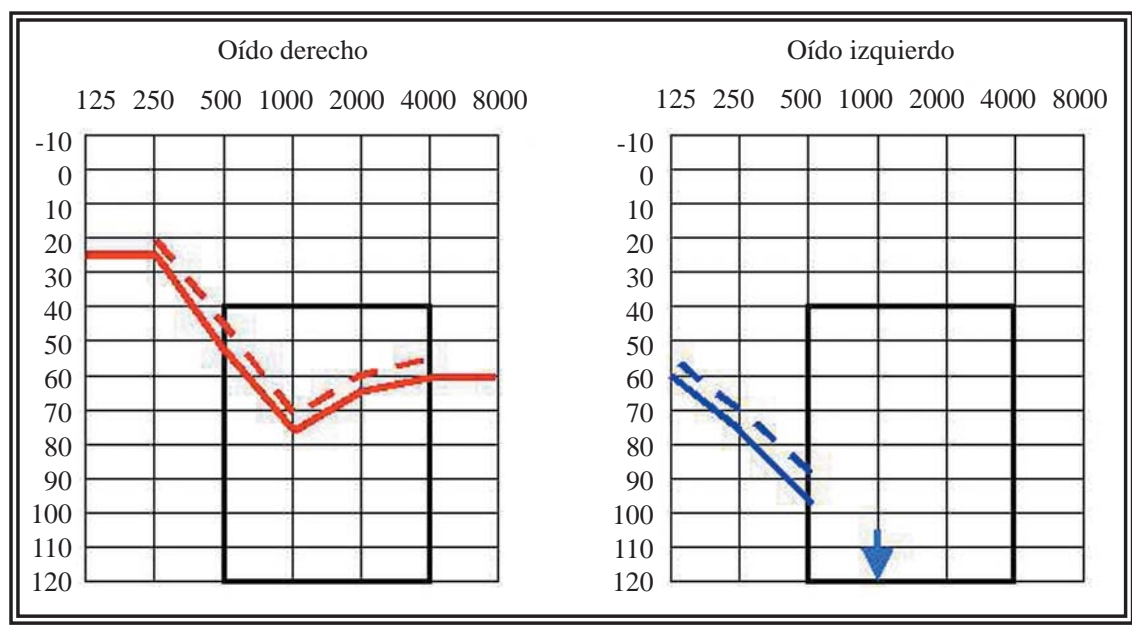

FIGURA 2. Audiometria a los 15 días Oído derecho: 76,5\% Oído izquierdo: 100\%

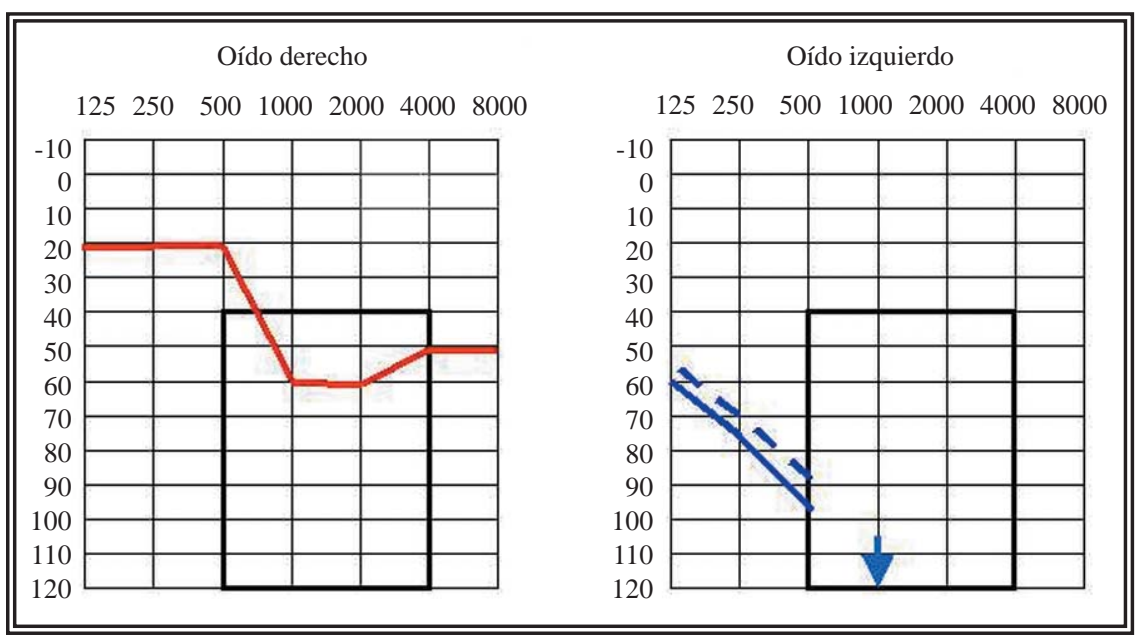

FIGURA 3. Audiometria a los 6 meses. Oido derecho 58,6\%. Oído izquierdo: 100\% (tablas AMA).

vestibular. Cuando aparecen alteraciones auditivas en un paciente bajo tratamiento es mandatario interrumpir la administración del medicamento, recuperando los niveles auditivos en las primeras $24 \mathrm{~h}$. La prevención puede realizarse con una administración lenta y monitorización plas- mática (mantener niveles $<50$ $\mathrm{mcg} / \mathrm{mL}$ ), evitando su asociación con otros fármacos ototóxi$\cos$ (antibióticos aminoglucósidos) y/o fraccionando la dosificación oral ${ }^{7}$.

Histológicamente se ha demostrado que producen alteraciones en la estría vascular de la cóclea, consistentes en edema intercelular, estrechamiento del calibre de los vasos y degeneración de la capa intermedia. Audiométricamente la ototoxicidad por diuréticos suele producir una pérdida bilateral, más o menos simétrica que afecta a todas las frecuencias: curva plana o en "U". Los PEATC y las OEA pueden ser útiles en la detección precoz de la ototoxicidad $^{9}$. No fueron empleadas en nuestro caso por tratarse de una hipoacusia profunda ya instaurada. El tratamiento consta fundamentalmente de la supresión del fármaco. Consideramos que dada la etiología el tratamiento corticoideo y el vasodilatador podrían ser de utilidad para reducir el edema de la estría vascularis.

\section{REFERENCIAS}

1. Wilson WR, Gulya AJ. Sudden Sensorineural Hearing Loss. In Cummings CW, Harker LA editors: Otolaryngology Head and Neck Surgery, 2nd ed. St. Louis, 1993

2. Martin GK, Jassir D, Stagner 88, Lonsbury-Martin BL. Effects of loop diuretics on the suppression tuning of distortion-product otoacoustic emissions in rabbits. J Acoust Soc Am. 1998;104(2 Pt 1):972-983.

Tabla 1. Fármacos con ototoxicidad demostrada.

\begin{tabular}{ll}
\hline DIURÉTICOS & Furosemida; Acido etacrinico; Bumetanida; Acetazolamida; Manitol; Clorotiazidas. \\
& Estreptomicina; Dihidroestreptomicina; Neomicina; Gentamicina; Kanamicina; \\
ANTIBIOTICOS y & Tobramicina; Amikacina; Sisomicina; Netilmicina; Dibekacina; Vancomicina; \\
AMINOGLUCÓSIDOS & Eritromicina; Cloranfenicol; Ristocetina; Polimixina B; Viomicina; Farmacetina; \\
& Colistina; Ampicilina; Cicloserina; Kanendomicina.
\end{tabular}

ANALGÉSICOS y ANTIPIRÉTICOS Aspirina; Salicilatos; Quinina; Cloroquina 
3. Mulheran M, Harpur ES, The effect of gentamicin and furosemide given in combination on cochlear potentials in the guinea pig. Br J Audiol. 1998;32(1):47-56.

4. Rais-Bahrami K, Majd M, Veszelovszky E, Short BL. Use of furosemide and hearing loss in neonatal intensive care survivors. Am J Perinatol. 2004;21(6):329-332.

5. Rybak LP, Whitworth C, Scott V, Weberg A. Ototoxicity of furosemide during development. Laryngoscope. 1991;101 (11): 1167-1174

6. Quick CA, Hoppe W. Permanent deafness associated with furosemide administration. Ann Otol Rhinol Laryngol 1975;84(1 Pt 1):94-101.

7. Rybak LP, Matz GJ: Effects of Toxic Agents. In Cummings CW, Harker LA editors: Otolaryngology Head and Neck Surgery, 2nd ed. St. Louis, 1993

8. Koegel L Jr. Ototoxicity: A contemporary review of Aminoglycosides, loop diuretics, acetylsalicylic acid, quinine, erythromycin and cisplatinum. Am J Otol. 1985;6(2):190-199.
9. Freeman S, Plotnik M, Elidan J, Sohmer H. Differencial Effect of Loop diuretic furosemide on short latency auditory and Vestibular Evoked Potentials. Am J Otol. 1999;20 (1):41-45.

10. Baune B, Kessler V, Patris S, Descamps V, Casalino E, Quenon JL, et al. Medicinal iatrogenics in hospitals. A survey on a given day. Presse Med. 2003;32(15):683-688.

Correspondencia autor: Dr. R.H. Martínez Rodríguez Servicio de Urología.

Fundació Puigvert.

Cartagena, 340-350. 08025 Barcelona

E-mail: hugomar2@yahoo.es

Información artículo: Nota clínica

Trabajo recibido: septiembre 2006

Trabajo aceptado: noviembre 2007 